\title{
Loss of CIC and FUBP1 expressions are potential markers of shorter time to recurrence in oligodendroglial tumors
}

\author{
Aden Ka-Yin Chan ${ }^{1,2}$, Jesse Chung-Sean Pang ${ }^{1,2}$, Nellie Yuk-Fei Chung ${ }^{1,2}$, \\ Kay Ka-Wai Li Li,2, Wai Sang Poon ${ }^{3}$, Danny Tat-Ming Chan ${ }^{3}$, Zhifeng Shi ${ }^{4}$, Liang Chen ${ }^{4}$, \\ Liangfu Zhou ${ }^{4}$ and Ho-Keung $\mathrm{Ng}^{1,2}$
}

${ }^{1}$ Department of Anatomical and Cellular Pathology, Chinese University of Hong Kong, Hong Kong, China; ${ }^{2}$ The Chinese University of Hong Kong-Shenzhen Research Institute, Shenzhen, China; ${ }^{3}$ Neurosurgery Division, Department of Surgery, Chinese University of Hong Kong, Hong Kong, China and ${ }^{4}$ Department of Neurosurgery, Huashan Hospital, Fudan University, Shanghai, China

\begin{abstract}
Combined deletion of chromosomes $1 p$ and $19 q$ is a prognostic marker in oligodendroglial tumors. Recent studies in oligodendroglial tumors have unveiled recurrent mutations of CIC (homolog of Drosophila capicua) and FUBP1 (far upstream element binding protein 1) that are located on 19q13 and 1p31, respectively. However, the impact of $C I C$ and FUBP1 mutations on their protein expressions has not been examined. The aims of this study were to correlate the expression patterns of CIC and FUBP1 with their mutation profiles and to evaluate the clinical relevance of these molecular markers in $\mathbf{5 5}$ oligodendroglial tumors diagnosed in $\mathbf{4 7}$ adult patients. Using direct sequencing, somatic mutations of $C I C$ and FUBP1 were identified in $47 \%(22 / 47)$ and $16 \%(7 / 45)$ of oligodendroglial tumors, respectively. Immunohistochemical analysis revealed loss of CIC or FUBP1 protein expression in $36 \%(20 / 55)$ and $16 \%(9 / 55)$ of oligodendroglial tumors examined. Somatic mutation was significantly associated with absent protein expression for both genes $(C I C, P=0.01 ; F U B P 1, P=0.00001)$. Four tumors with undetectable $C I C$ mutations exhibited absent $\mathrm{CIC}$ expression, suggesting that $\mathrm{CIC}$ inactivation could be mediated by mechanisms other than mutations and genomic loss. Univariate survival analysis revealed that $1 \mathrm{p} / 19 \mathrm{q}$ codeletion was significantly associated with overall survival $(P=0.05)$. Loss of $\mathrm{CIC}$ expression was significantly correlated with shorter progression-free survival $(P=0.03)$, whereas $C I C$ alteration (mutation or null expression) with worse overall survival $(P=0.05)$. Absent FUBP1 expression was linked with unfavorable progression-free survival $(P=0.02)$ and overall survival $(P=0.01)$. In 16 tumors with $1 p / 19 q$ codeletion, $C I C$ mutation was associated with unfavorable survival $(P=0.01)$. There was a correlation between lack of CIC or FUBP1 expression and poor progression-free survival $(P=0.004 ; P=0.0003)$. No molecular markers showed association with survival in oligodendroglial tumors lacking $1 p / 19 q$ codeletion. We conclude that absent $\mathrm{CIC}$ and FUBP1 expressions are potential markers of shorter time to recurrence and $\mathrm{CIC}$ mutation a potential marker of worse prognosis, especially in tumors carrying $1 \mathrm{p} / 19 \mathrm{q}$ codeletion.
\end{abstract}

Modern Pathology (2014) 27, 332-342; doi:10.1038/modpathol.2013.165; published online 13 September 2013

Keywords: CIC; FUBP1; immunohistochemistry; mutation; oligoastrocytoma; oligodendroglioma

Oligodendroglial tumors, comprising oligodendroglioma and oligoastrocytoma, are primary brain neoplasms accounting for $5-20 \%$ of all gliomas. Unlike other glioma subtypes of similar grade, oligodendroglial tumors exhibit better patient survi-

Correspondence: Dr AK-Y Chan, MBChB or Professor H-K Ng, MBChB(Edin), MD, FRCPath, FRCPA, Department of Anatomical and Cellular Pathology, The Chinese University of Hong Kong, 30-32 Ngan Shing Street, Shatin, Hong Kong, China.

E-mail: adenchan@cuhk.edu.hk or hkng@cuhk.edu.hk

Received 3 April 2013; accepted 29 July 2013; published online 13 September 2013 val and sensitivity to chemotherapy containing procarbazine, lomustine and vincristine. ${ }^{1}$ In spite of these favorable inherent behaviors, most oligodendroglial tumors recur eventually, with some of them even progressing to higher grade lesion. Genetically, oligodendroglial tumors are characterized by a combined loss of chromosome arms $1 p$ and 19q and mutations in the isocitrate dehydrogenase (IDH) genes. The $1 \mathrm{p} / 19 \mathrm{q}$ codeletion signature is associated with classic oligodendroglial histology, longer survival and sensitivity to chemotherapy, ${ }^{2-4}$ and has been demonstrated as a predictor of favorable prognosis and treatment response in randomized 
clinical trials. ${ }^{5,6}$ The IDH mutation is thought to be a very early event in gliomagenesis and is a positive prognostic marker in oligodendroglial tumors. ${ }^{5,7-9}$

Studies have suggested that $1 \mathrm{p} / 19 \mathrm{q}$ codeletion is mediated by an unbalanced translocation between chromosomes 1 and 19, generating two derivative chromosomes $\operatorname{der}(1 ; 19)(q 10 ; p 10)$ and $\operatorname{der}(1 ; 19)$ (p10;q10), with the latter chromosome being lost (resulting in the $1 \mathrm{p} / 19 \mathrm{q}$ codeletion genotype) during tumor development. ${ }^{10,11}$ The target gene(s) underlying $1 p / 19 q$ codeletion or $t(1 ; 19)(q 10 ; p 10)$ translocation remains elusive. Recent next-generation sequencing analysis of oligodendroglial tumors has unveiled inactivating mutations in the CIC (homolog of Drosophila capicua) and FUBP1 (far upstream element binding protein 1) genes, which are respectively mapped to $19 q 13.2$ and $1 p 31.1$, in 1p/19q-codeleted oligodendroglioma. ${ }^{12}$ An independent study using a similar approach revealed the presence of $C I C$ mutation but not FUBP1 mutation in oligodendroglioma. ${ }^{13}$ Subsequent studies confirmed the presence of $C I C$ and FUBP1 mutations in oligodendroglioma as well as in oligoastrocytoma. ${ }^{14,15}$ In contrast, such mutations were rarely found in astrocytoma and glioblastoma. ${ }^{13-15}$ CIC and FUBP1 mutations were strongly associated with $1 \mathrm{p} / 19 \mathrm{q}$ codeletion, suggesting that chromosomal loss of $1 \mathrm{p}$ and $19 \mathrm{q}$ might contribute to inactivation of these genes. The role $C I C$ and FUBP1 has in oligodendroglial tumor development is unclear.

Considering that a larger proportion of CIC and FUBP1 mutations identified were predicted to be detrimental to CIC and FUBP1 structure or functions, ${ }^{12-15}$ the impact of these mutations on protein expression has not been examined. Moreover, determining protein expression may uncover additional inactivated $C I C$ and FUBP1 not mediated by mutations. The aims of this study were to correlate the expression patterns of CIC and FUBP1 with their mutation profiles and to evaluate the clinical relevance of these molecular markers in a series of 55 oligodendroglial tumors diagnosed in 47 adult patients. Our results demonstrated that mutation and null expression of CIC and FUBP1 were detectable in $55 \%$ and $18 \%$ of oligodendroglial tumors, respectively. Somatic mutations were correlated with absent expressions for both genes. In 1p/19q-codeleted tumors, lack of CIC or FUBP1 expression was significantly associated with shorter time to recurrence, whereas the presence of CIC mutation was correlated with unfavorable outcome.

\section{Materials and methods}

\section{Patients and Tissue Specimens}

Gliomas diagnosed between 1994 and 2009 at the Department of Anatomical and Cellular Pathology, Prince of Wales Hospital (Hong Kong, China) were reviewed. A total of 55 oligodendroglial tumors diagnosed in 47 adult patients were recruited for this study. The tumors, classified according to the current World Health Organization (WHO) criteria, ${ }^{16}$ comprised 19 oligodendrogliomas (WHO grade II; OII), 11 anaplastic oligodendrogliomas (WHO grade III; OIII), 18 oligoastrocytomas (WHO grade II; OAII) and 7 anaplastic oligoastrocytomas (WHO grade III; OAIII) (Supplementary Table S1). There were eight tumor pairs with primary and subsequent recurrent tumors (O6, O16, O17, O34, OA3, OA13, OA21 and OA30) and seven cases with recurrent tumors but no paired primary tumor $(\mathrm{O} 2 \mathrm{r}, \mathrm{O} 7 \mathrm{r}, \mathrm{O} 10 \mathrm{r}, \mathrm{O} 13 \mathrm{r}, \mathrm{O} 18 \mathrm{r}$, O25r and O26r). Formalin-fixed, paraffin-embedded tumor material was used in this study. Ten samples with insufficient tissue material were subjected to fewer molecular analyses. Patient demographics, clinical and follow-up data were retrieved from the institutional electronic medical record system. Tumormatched blood samples were available for 43 patients and served as constitutional controls. This study was approved by the New Territories East Cluster-Chinese University of Hong Kong ethics committee.

\section{Mutation Analysis of CIC, FUBP1, IDH1 and IDH2}

Tissues from representative areas with tumor content $>70 \%$ were collected from 5 to 7 dewaxed formalinfixed, paraffin-embedded sections and treated with proteinase $\mathrm{K}$ at a final concentration of $2 \mu \mathrm{g} / \mu \mathrm{l}$ in $10 \mathrm{mM}$ Tris-HCl buffer $(\mathrm{pH} 8.5)$ at $55^{\circ} \mathrm{C}$ for $2-18 \mathrm{~h}$ and then at $98^{\circ} \mathrm{C}$ for $10 \mathrm{~min}$. After centrifugation, the supernatant containing the crude cell lysate was used in subsequent PCR analysis. DNA was amplified in a $10-\mu \mathrm{l}$ reaction volume containing $1-2 \mu \mathrm{l}$ of cell lysate, $10 \mathrm{mM}$ Tris-HCl (pH 8.3), $50 \mathrm{mM}$ KCl, $2.5 \mathrm{mM}$ $\mathrm{MgCl}_{2}, 0.2 \mathrm{mM}$ of each deoxyribonucleoside triphosphate, $0.4 \mathrm{mM}$ of each primer and $0.2 \mathrm{U}$ of AmpliTaq Gold DNA polymerase (Life Technologies Corporation, Hong Kong, China). PCR conditions included $95^{\circ} \mathrm{C}$ for $10 \mathrm{~min}, 45-50$ cycles of $95^{\circ} \mathrm{C}$ for $20 \mathrm{~s}, 60-$ $66^{\circ} \mathrm{C}$ for $20 \mathrm{~s}$ and $72{ }^{\circ} \mathrm{C}$ for $30 \mathrm{~s}$, and a final extension step of $72^{\circ} \mathrm{C}$ for $3 \mathrm{~min}$. Products were then treated with exonuclease I and thermosensitive alkaline phosphatase (TakaRa Biotechnology Limited, Dalian, China). Sequencing was performed using BigDye Terminator Cycle Sequencing kit v.1.1 (Life Technologies). The products were resolved in the Genetic Analyzer 3130xl and analyzed by Sequencing Analysis software (Applied Biosystems, Life Technologies Corporation, NY, USA). All base changes were confirmed in newly prepared cell lysates. Mutations were considered somatic when not detected in corresponding tumor-matched blood samples. Primer sequences are listed in Supplementary Table S2.

\section{1p/19q Status by Fluorescence In Situ Hybridization}

The $1 p / 19 q$ codeletion status was evaluated by dual-color fluorescence in situ hybridization (FISH) analysis. ${ }^{17}$ Briefly, 5- $\mu$ m-thick formalin-fixed, 
paraffin-embedded sections were deparaffinized, treated with sodium thiocyanate and digested in pepsin solution. Locus-specific probes were generated from bacterial artificial chromosome clones by nick translation, in the presence of SpectrumOrangedeoxyuridine triphosphate (dUTP) (Abbott Laboratories, Hong Kong, China) or digoxigenin-dUTP (Roche Diagnostics, Hong Kong, China). The labeled probes mixed with Cot-1 DNA (Life Technologies) were denatured and hybridized to sections, which were then washed and incubated with antidigoxigenin antibody conjugated with fluorescein isothiocyanate. Sections were mounted with Vectashield mounting medium containing 4',6-diamidino2-phenylindole (Vector Laboratories, Burlingame, CA, USA) and viewed under a Zeiss Axioplan fluorescence microscope (Carl Zeiss Microscopy LLC, NY, USA). Hybridizing signals in at least 100 non-overlapping nuclei were counted. The loci interrogated were 1p36.3 (RP11-62M23 labeled red)/ 1q25.3-q31.1 (RP11-162L13 labeled green) and 19q13.3 (CTD-2571L23 labeled red)/19p12 (RP11$420 \mathrm{~K} 14$ labeled green). A sample was considered $1 \mathrm{p}$ or $19 \mathrm{q}$ deleted when more than $50 \%$ of counted nuclei exhibited one target (red) signal and two reference (green) signals.

\section{Expression of CIC and FUBP1}

Immunohistochemistry was used to determine expressions of CIC and FUBP1. Briefly, dewaxed formalin-fixed, paraffin-embedded slides were subjected to antigen retrieval by heating in Tris-EDTA buffer containing $10 \mathrm{mM}$ Tris-HCl (pH 9.0), $1 \mathrm{mM}$ ethylenediaminetetraacetic acid and $0.05 \%$ Tween20. After antigen retrieval, the slides were processed by BenchMark XT automated tissue staining systems (Ventana Medical Systems, Tucson, AZ, USA) using validated protocols. Tissue sections were incubated at $37^{\circ} \mathrm{C}$ for $32 \mathrm{~min}$ with primary antibody, followed by incubation with UltraView HRP-conjugated multimer antibody reagent (Ventana Medical Systems). Antigen detection was performed using UltraView diaminobenzidine chromogen step (Ventana Medical Systems). Tissues were counterstained with Mayer's hematoxylin and mounted for scoring. The antibodies used were rabbit anti-human CIC antibody (1:100 dilution; LS-B4752; Lifespan Biosciences, Seattle, WA, USA), which was raised against amino-acid residues 1500-1608 mapped to the $\mathrm{C}$ terminus of human CIC, and goat anti-human FUBP1 antibody (C-20; 1:100 dilution; Santa Cruz Biotechnology, Santa Cruz, CA, USA), which recognizes the $\mathrm{C}$ terminus of human FUBP1. Sections without primary antibody treatment served as negative control. Protein expression was reviewed independently by two authors (AKYC and JCSP) blinded to the clinical data. Cases that differed significantly between readers were re-evaluated to reach a consensus. Expressions of CIC and FUBP1 were evaluated for nuclear staining pattern and scored semiquantitatively by multiplying the degree of intensity by percentage of stained cells. Intensity was graded as 0 (no staining), 1 (weak), 2 (moderate) and 3 (strong), whereas the percentage of stained cells as $0(<10 \%), 1(10-50 \%)$ and $2(>50 \%)$. The score was interpreted as absent $(0)$, weak $(1,2)$, moderate $(3,4)$ or strong $(6)$.

\section{Statistical Analysis}

Statistical analysis was conducted using the SPSS version 18 software (IBM Corporation, New York, NY, USA). The correlations between molecular markers and clinical factors were evaluated by $\chi^{2}$ test or Fisher's exact test, whichever was appropriate. Differences between groups were assessed by Student's $T$-test or Mann-Whitney $U$-test. Kaplan-Meier curve was used to determine survival distribution and logrank test to compare differences between two groups. Multivariate analysis was assessed with the Cox proportional hazards regression model. Overall survival (OS) was defined as the time of diagnosis until death or last follow-up, whereas progression-free survival (PFS) was the time from initial surgery until tumor recurrence or progression. A $P$-value $<0.05$ (two-sided) was considered statistically significant.

\section{Results}

\section{CIC Mutations}

Forty-seven tumors were assessed for mutations in all 20 coding exons and exon-intron boundaries of the CIC gene by direct DNA sequencing. Twentythree base changes in CIC were identified in 15 primary and 7 recurrent tumors, with O10r harboring two mutations (Table 1 and Supplementary Figures S1 and S2). These base alterations were not found in the corresponding tumor-matched blood DNA, indicating that they were somatically acquired. The mutations were detected in 8 of 17 (47\%) oligodendrogliomas, 9 of $11(82 \%)$ anaplastic oligodendrogliomas, 3 of $13(23 \%)$ oligoastrocytomas and 2 of 6 $(33 \%)$ anaplastic oligoastrocytomas. CIC mutation was significantly associated with tumors with classic oligodendroglial histology $(P=0.04)$. There were 13 insertion/deletions (indels), 9 missense mutations and 1 nonsense mutation. The missense mutations clustered at two functional domains: the DNAbinding domain named the high-mobility group (HMG) box, corresponding to codons 200-268 on exon 5 and part of exon 6 , and the C1 motif with repressor activity, corresponding to exon 20. Using PolyPhen2, all five substitution mutations at the HMG box and two of four mutations at C1 motif were predicted to have damaging effects on CIC structure or function. ${ }^{18}$ It was previously demonstrated that substitution mutation R505W (corresponding to R215W in human CIC) at the HMG box led to 
Table 1 Somatic mutations and immunohistochemical expression patterns of CIC and FUBP1 in oligodendroglial tumors

\begin{tabular}{|c|c|c|c|c|c|c|c|c|}
\hline \multirow[b]{2}{*}{ Case no. } & \multirow[b]{2}{*}{$\begin{array}{l}\text { Tumor type } \\
\text { and grade }\end{array}$} & \multicolumn{3}{|c|}{ Mutations } & \multirow[b]{2}{*}{ Exon } & \multirow[b]{2}{*}{$\begin{array}{l}\text { CIC IHC } \\
\text { expression }\end{array}$} & \multirow[b]{2}{*}{$\begin{array}{l}\text { FUBP1 IHC } \\
\text { expression }\end{array}$} & \multirow[b]{2}{*}{$\begin{array}{c}1 p / 19 q \\
\text { Codeletion }\end{array}$} \\
\hline & & Nucleotide change & Protein change & $\begin{array}{l}\text { Mutation } \\
\text { type }\end{array}$ & & & & \\
\hline \multicolumn{9}{|l|}{ CIC } \\
\hline O6r & OII & c. $794 \mathrm{C}>\mathrm{T}$ & p.Pro265Leu & Missense & 6 & ++ & +++ & Yes \\
\hline O16* & OII & c. $2981 \mathrm{delA}$ & p.Gln $994 A \operatorname{Argfs}^{*} 45$ & Deletion & 12 & No & No & Yes \\
\hline $\mathrm{O} 17$ & OII & c.630_632delCAG & p.Ser211del & Deletion & 5 & No & ++ & Yes \\
\hline $\mathrm{O} 17 \mathrm{r}$ & OII & c.633delC & p.Ser211Argfs* 46 & Deletion & 5 & No & ++ & Yes \\
\hline O19 & OII & c. $3640 \mathrm{C}>\mathrm{T}$ & p.Arg1214X & Nonsense & 15 & No & No & Yes \\
\hline $\mathrm{O} 24^{*}$ & OII & c. $797 \mathrm{~A}>\mathrm{T}$ & p.Asp266Val & Missense & 6 & ++ & + & Yes \\
\hline O31 & OII & c. $4784 \mathrm{C}>\mathrm{T}$ & p.Ser1595Phe & Missense & 20 & ++ & +++ & No \\
\hline O34 & OII & c. $643 \mathrm{C}>\mathrm{T}$ & p.Arg215Trp & Missense & 5 & No & +++ & No \\
\hline O10r & OIII & с.613_614delAA; c.616delG & p.Asn205_Ala206delinsSer & Deletion & 5 & ++ & + & No \\
\hline O11* & OIII & c. $607 \mathrm{C}>\mathrm{G}$ & p.Pro203Ala & Missense & 5 & No & No & No \\
\hline $\mathrm{O} 13 \mathrm{r}$ & OIII & c.2694_2695insC & p.Lys899Glnfs* 32 & Insertion & 10 & ++ & +++ & No \\
\hline O16r* & OIII & c. 2981 delA & p.Gln994Argfs* 45 & Deletion & 12 & No & No & Yes \\
\hline $\mathrm{O} 23$ & OIII & c. $4420 \quad 4422$ delGTC & p.Val1474del & Deletion & 19 & No & +++ & No \\
\hline $\mathrm{O} 32$ & OIII & c. $4643 \mathrm{C}>\mathrm{T}$ & p.Ala1548Val & Missense & 20 & ++ & +++ & Yes \\
\hline $\mathrm{O} 33$ & OIII & c.4116delC & p.Pro1372fs* 43 & Deletion & 17 & + & +++ & Yes \\
\hline O34r & OIII & c. $643 \mathrm{C}>\mathrm{T}$ & p.Arg215Trp & Missense & 5 & No & +++ & Yes \\
\hline O39* & OIII & c. $4543 \mathrm{C}>\mathrm{T}$ & p.Arg1515Cys & Missense & 20 & No & No & Yes \\
\hline OA15 & OAII & c. $4790 \mathrm{delC}$ & p.Pro1597fs & Deletion & 20 & +++ & +++ & No \\
\hline OA23 & OAII & c. 2017 2018delCC & p.Thr673Lysfs * 16 & Deletion & 10 & No & +++ & Yes \\
\hline OA $25^{*}$ & OAII & c. $4 \overline{7} 90 \mathrm{delC}$ & p.Pro1597fs & Deletion & 20 & No & No & Yes \\
\hline OA6 & OAIII & c. $4781 \mathrm{C}>\mathrm{T}$ & p.Pro1594Leu & Missense & 20 & ++ & +++ & No \\
\hline OA21r & OAIII & c.768_770delGAA & p.Lys257del & Deletion & 6 & ++ & +++ & No \\
\hline \multicolumn{9}{|l|}{ FUBP1 } \\
\hline $\mathrm{O} 24^{*}$ & OII & c. $343+1$ delG & Splice donor site & Splicing & 5 & ++ & + & Yes \\
\hline $\mathrm{O} 16^{*}$ & OII & c. $416-1 \mathrm{G}>\mathrm{C}$ & Splice acceptor site & Splicing & 7 & No & No & Yes \\
\hline O16r* & OIII & c. $416-1 \mathrm{G}>\mathrm{C}$ & Splice acceptor site & Splicing & 7 & No & No & Yes \\
\hline O39* & OIII & c.622_623insATTA & p.Ile208Asnfs* 3 & Insertion & 8 & No & No & Yes \\
\hline $\mathrm{O} 11^{*}$ & OIII & c.1374_1398delACCCCATGGCCCCCAT & p.Val458Alafs* 24 & Deletion & 15 & No & No & No \\
\hline $\mathrm{O} 27$ & OIII & C. $1551 \mathrm{G}>\mathrm{A}$ & p.Trp517X & Nonsense & 16 & + & No & Yes \\
\hline OA $25^{*}$ & OAII & c.120delG/c.120 + 1delG & $\begin{array}{c}\text { p.Gln } 40 f^{*} 14 / \text { splice donor } \\
\text { site }\end{array}$ & $\begin{array}{l}\text { Deletion/ } \\
\text { splicing }\end{array}$ & 1 & No & No & Yes \\
\hline
\end{tabular}

Abbreviations: OII, oligodendroglioma WHO grade II; OIII, oligodendroglioma WHO grade III; OAII, oligoastrocytoma WHO grade II; OAIII, oligoastrocytoma WHO grade III; +, weak expression; ++ , moderate expression; +++ , strong expression.

Case number with suffix $\mathrm{r}$ indicates recurrence and with asterisk represents mutations in both CIC and FUBP1. Reference cDNA sequence for CIC is NM_015125 and for FUBP1 NM_003902.

impaired eye development in Drosophila. ${ }^{19}$ Moreover, the indels were predominantly of frameshift and small inframe deletions at functional domains including the HMG box and the CI motif that are predicted to disrupt CIC function. The mutation profile suggests that CIC function is likely compromised in oligodendroglial tumors harboring CIC mutations. Four of eight recurrent pairs were found to harbor CIC mutations. $\mathrm{O} 16$ and $\mathrm{O} 34$ displayed identical mutations in primary and recurrent tumors. $\mathrm{O} 17$ had a 3-bp deletion at exon 5 and its recurrent sample $\mathrm{O} 17 \mathrm{r}$ a 1-bp deletion at the same exon, supporting the notion that the HMG box is a susceptible site for mutation. Case OA21 carried wild-type CIC in primary tumor but acquired mutation at exon 6 upon progression to higher grade.

Of 22 tumors with CIC mutations, 9 had no 1p/19q codeletion. Two tumors (O34 and O11) carrying missense mutations at exon 5 showed a single mutant peak in the sequencing electropherogram, suggesting that $\mathrm{O} 34$ and $\mathrm{O} 11$ might harbor partial deletion at the CIC locus or contained identical double mutations. The latter event is infrequently seen in human cancer. The remaining seven tumors exhibited heterozygous peaks for missense mutations or overlapping sequencing peaks after indels, consistent with a genotype of balanced 19q. Taken together, 15 of $22(68 \%)$ tumors with CIC mutations had genomic loss on the non-mutated CIC allele.

\section{FUBP1 Mutations}

Forty-five tumors were examined for base changes in all 20 coding exons and exon-intron boundaries of the FUBP1 gene. Seven somatic FUBP1 mutations were detected in six primary and one recurrent tumors, representing two oligodendrogliomas, four anaplastic oligodendrogliomas and one oligoastrocytomas (Table 1 and Supplementary Figures S1 and S2)). There were three indels, one nonsense mutation and three splicing alterations. Case OA25 had a single base deletion at the exon 1/intron 1 boundary that was predicted to result in a frameshift deletion 

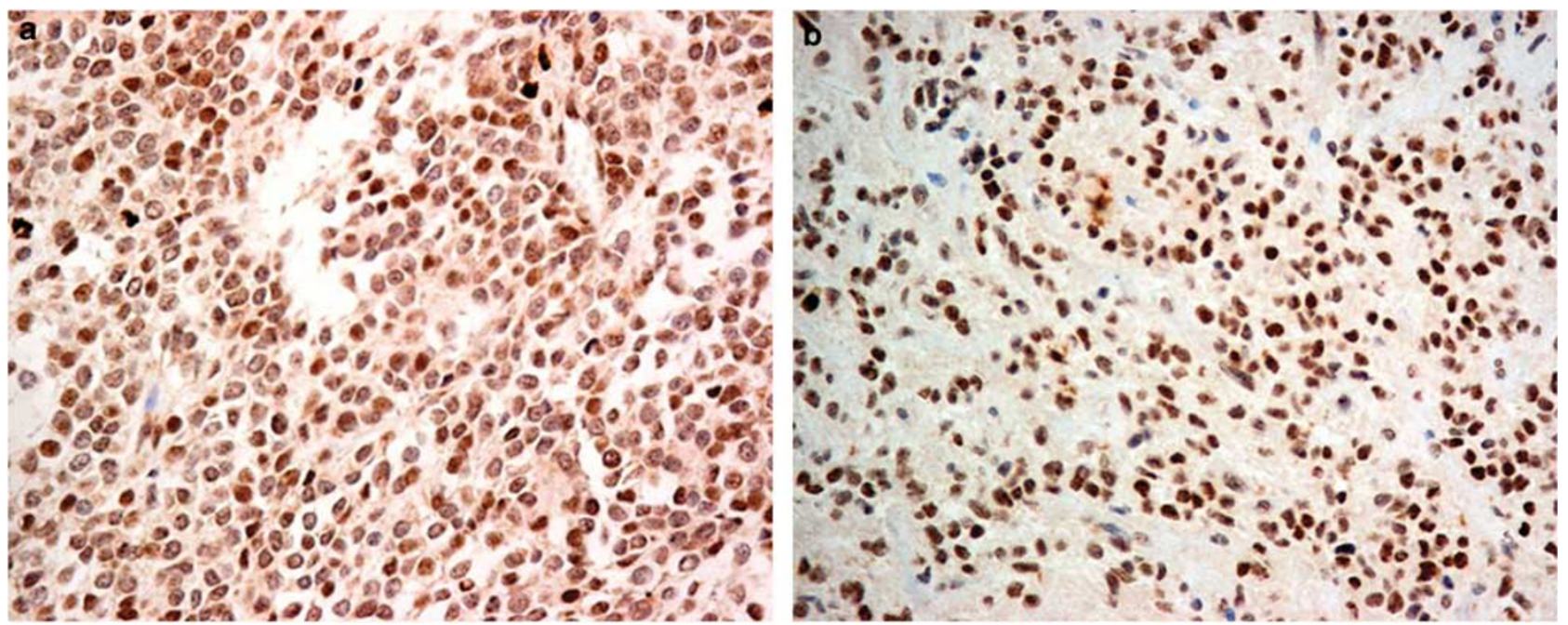

Figure 1 Representative images of immunohistochemical staining for CIC (homolog of Drosophila capicua) and FUBP1 (far upstream element binding protein 1) in oligodendroglial tumors. (a) Positive CIC expression with strong nuclear staining in anaplastic oligodendroglioma O13r $(\times 400)$. (b) Positive FUBP1 expression with strong nuclear staining in anaplastic oligoastrocytoma OA6 $(\times 400)$.

or a splicing aberration. Case O16 displayed identical FUBP1 mutation at the splice site in primary and recurrent tumors. Six of seven FUBP1-mutated tumors concurrently carried CIC mutations and a significant association was observed $(P=0.04)$. No association was found between FUBP1 mutation and $1 \mathrm{p} / 19 \mathrm{q}$ status.

\section{CIC Expression}

To better understand the involvement of CIC in oligodendroglial tumors, we determined its expression pattern by immunohistochemistry in 55 oligodendroglial tumors. We summarized the CIC mutation distribution from our data and literature and found $>93 \%$ of mutations occurred at positions before exon 20 . We therefore used an antibody that recognizes the $\mathrm{C}$ terminus of CIC in immunohistochemistry. Absent, weak, moderate and strong CIC expression was detected in 20 (36\%), 5 (9\%), 26 $(47 \%)$ and $4(7 \%)$ cases, respectively (Supplementary Table S1 and Figures 1 and 2). In 13 1p/19qcodeleted tumors harboring CIC mutation, 9 (69\%) showed absent CIC expression, 1 weak expression and 3 moderate expression. The latter three cases harbored missense mutations, which might not have affected the epitope recognized by the antibody. Tumors $\mathrm{O} 34$ and $\mathrm{O} 11$ carrying CIC mutation expressed no CIC, consistent with our speculation that these tumors harbored partial deletion of CIC. We also identified four tumors (O14, OA2, OA3 and OA9) with wild-type CIC sequence exhibiting CIC deficiency. Loss of CIC expression may be explained by the presence of second mutations in other parts of the CIC gene not examined or by mechanisms other than mutation and genomic loss.

Six cases with CIC mutation displayed moderate to strong CIC expression and all of them lacked 1p/19q codeletion. The frequency of CIC mutation identified in oligodendroglial tumors lacking $1 \mathrm{p} / 19 \mathrm{q}$ codeletion appeared to be higher than that reported. ${ }^{12-15}$ In five of six tumors, the mutations were either missense or small inframe deletions. It is possible that the antibody we used could interact with such mutant proteins. This speculation is supported by the detection of CIC mutant protein by western blotting in 1p/19q-codeleted oligodendroglial tumor cell lines carrying CIC mutations. ${ }^{13}$ Alternatively, the possibility of CIC translated from the non-mutated allele cannot be excluded.

Regarding the eight paired tumors, three (O16, O17 and O34) showed absent CIC expression in primary tumors and recurrences, and two (O6, OA30) had expression in primary and recurrent tumors. Three pairs (OA3, OA13 and OA21) exhibited loss of CIC expression in primary tumors but expressed CIC in recurrences. Tumor OA21 and its recurrence OA21r shared a genotype of IDH2 mutation and balanced $1 \mathrm{p} / 19 \mathrm{q}$, but the recurrent tumor showed some distinct features including higher tumor grade, tumor location (cerebellum vs frontal) and CIC expression. These results suggested that absent CIC expression identified in initial tumors might be a potential indicator for recurrence in oligodendroglial tumors. In total, 22 primary and 8 recurrent tumors exhibited $C I C$ alterations (either CIC mutation or absent CIC expression).

In correlation analysis, the four-tiered expression system for CIC was found not associated with any molecular markers. However, when CIC expression was scored as absent vs positive (including weak, moderate and strong expression), loss of expression was significantly associated with CIC mutation $(P=0.01)$ 
All cases
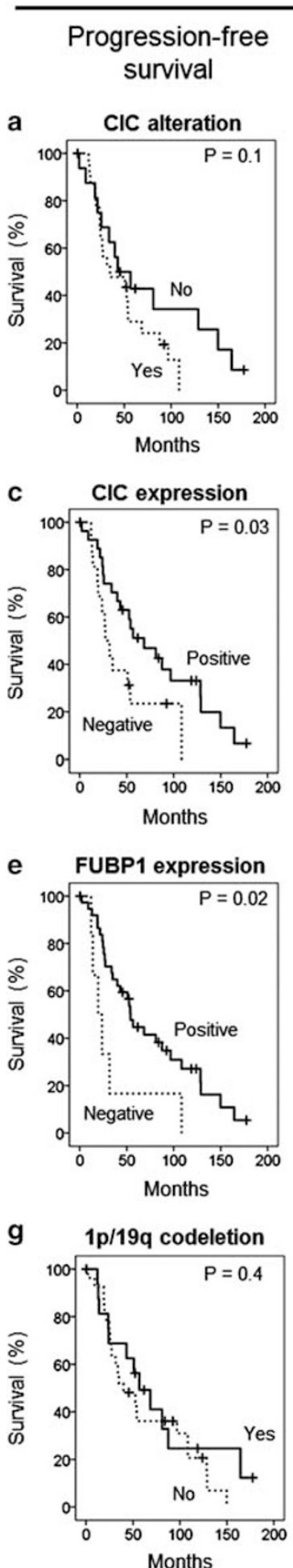

Overall survival
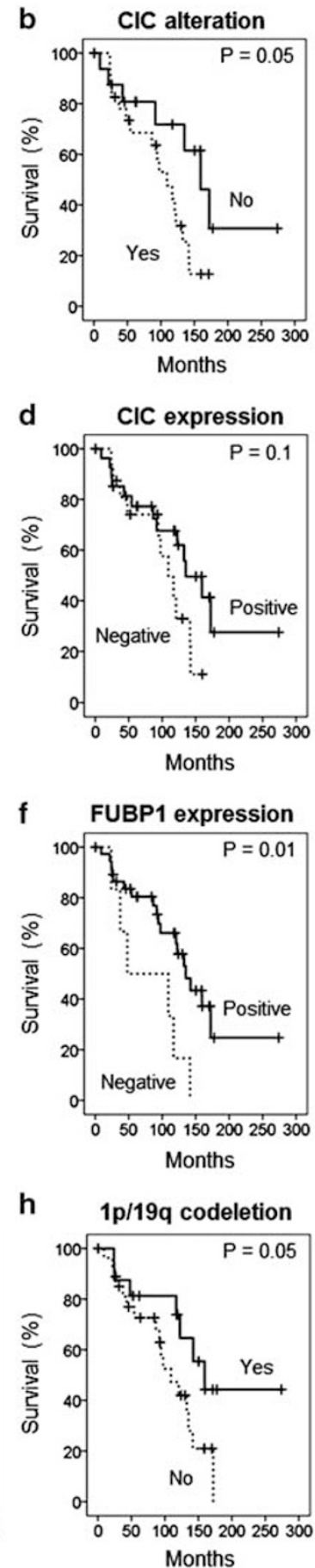

IDH mutated and $1 \mathrm{p} / 19 \mathrm{q}$ codeleted oligodendroglial tumors

$\begin{array}{cc}\begin{array}{c}\text { Progression-free } \\ \text { survival }\end{array} & \text { Overall survival }\end{array}$
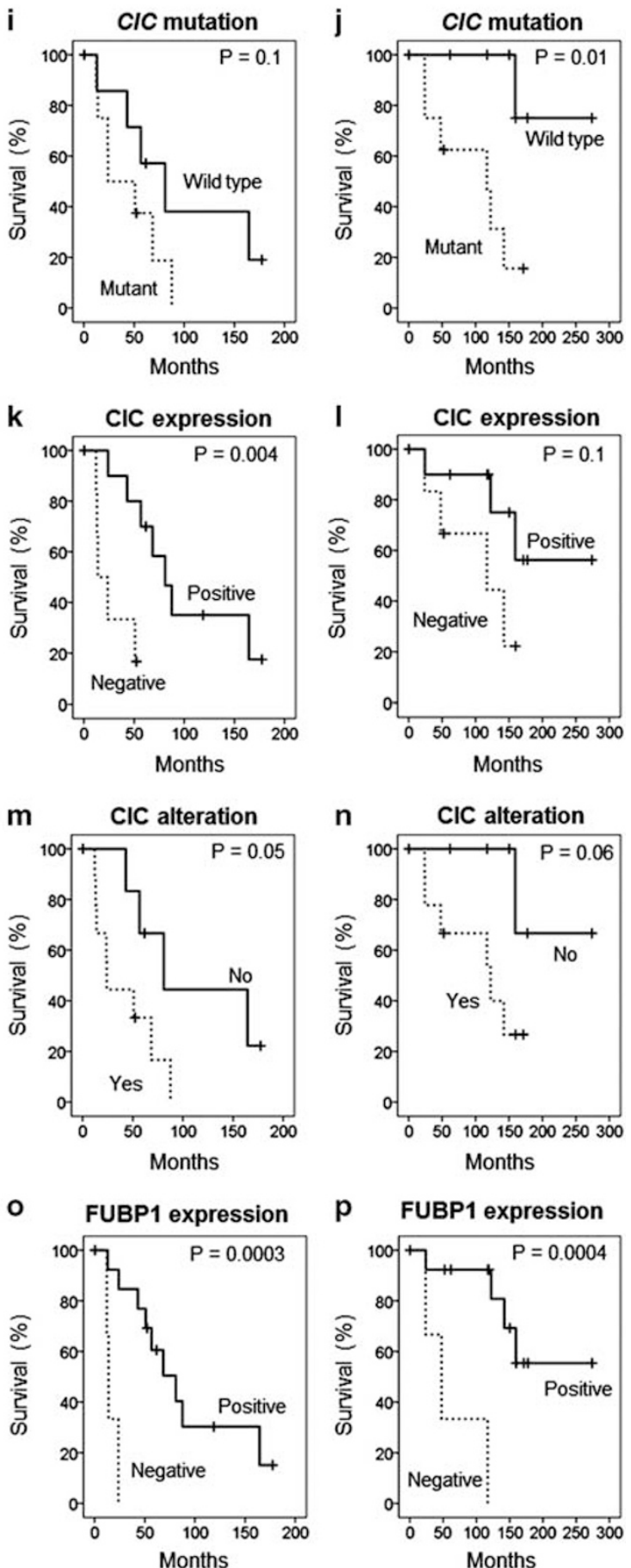

Figure 2 Kaplan-Meier curves for progression-free survival (PFS) and overall survival (OS) of molecular variables in oligodendroglial tumors. For all cases, CIC alteration is associated with OS (b) but not with PFS (a); CIC expression is correlated with PFS (c) but not with OS (d); FUBP1 expression is associated with both PFS (e) and OS (f); 1p/19q codeletion is correlated with OS (h) but not PFS (g). For 1p/ 19q-codeleted tumors, CIC mutation is associated with OS (j) but not PFS (i); CIC expression is correlated with PFS (k) but not OS (l); CIC alteration is associated with PFS (m) but not OS (n); FUBP1 expression is correlated with PFS (o) and OS (p). 


\section{FUBP1 Expression}

FUBP1 expression was examined by immunohistochemistry in 55 oligodendroglial tumors. Our result showed that FUBP1 expression was undetectable in eight primary and one recurrent tumors, but was present at weak level in $4(7 \%)$ cases, moderate in 11 $(20 \%)$ tumors and strong in 31 (56\%) samples. In six 1p/19q-codeleted tumors carrying FUBP1 mutation, five showed FUBP1 deficiency and one weak expression. In paired tumors, two (OA13 and OA21) showed FUBP1 deficiency in primary tumors but positive expression in recurrences, one (O16) exhibited null expression in both primary and recurrent tumors and five displayed expression in both primary tumors and recurrences. Taken together, nine primary and one recurrent tumors showed FUBP1 alterations (either FUBP1 mutation or absent FUBP1 expression). Except for one case, loss of FUBP1 expression was found concurrently with absent CIC expression $(P=0.001)$. Loss of FUBP1 expression (vs weak/moderate/strong expression) was significantly correlated with FUBP1 mutation $(P=0.00001)$ and $C I C$ mutation $(P=0.04)$.

\section{1p/19q Codeletion}

1p/19q Codeletion was detectable in 12 of $19(63 \%)$ oligodendrogliomas, 7 of 11 (64\%) anaplastic oligodendrogliomas, 5 of 18 (28\%) oligoastrocytomas and none in 7 anaplastic oligoastrocytomas examined, representing a codeletion rate of $44 \%$ in the series. Loss of $1 \mathrm{p} / 19 \mathrm{q}$ was associated with tumors with classic oligodendroglial histology $(P=0.001)$.

\section{IDH Mutations}

The IDH mutational hotspots, involving arginine at codon 132 of IDH1 and arginine at codon 172 of $I D H 2$, were evaluated by direct DNA sequencing in all samples. IDH mutations were detected in 18 of $19(95 \%)$ oligodendrogliomas, 11 of $11(100 \%)$ anaplastic oligodendrogliomas, 15 of 18 (83\%) oligoastrocytomas and 6 of 7 (86\%) anaplastic oligoastrocytomas, with an overall $I D H$ mutation frequency of $91 \%(50 / 55)$. No concurrent IDH1 and IDH2 mutations were detected. Except for case OA6, all tumors harboring $C I C$ or FUBP1 mutation had concomitant IDH mutation. All 1p/19q-codeleted tumors carried $I D H$ mutation, but only a trend was observed between these markers $(P=0.06)$.

\section{Survival Analysis}

Follow-up data were available for 44 patients. The median follow-up, median progression-free survival and median overall survival were 159 months (111-206 months), 53 months (35-70 months), and 133 months (111-154 months), respectively. At last follow-up, 25 patients had died, 16 were alive and 3 lost to follow-up.

Univariate analysis revealed that patients age $\leq 45$ years exhibited favorable progression-free survival $(P=0.00002)$ and overall survival $(P=0.002)$ and lower histologic tumor grade showed longer overall survival $(P=0.005)$ (Table 2). Regarding molecular markers, $1 p / 19 q$ codeletion was associated with longer overall survival $(P=0.05)$. Tumors with absent CIC expression showed shorter progressionfree survival than those expressing CIC $(P=0.03)$. There was a correlation between CIC alteration and worse survival $(P=0.05)$. For FUBP1, absent expression was linked with shorter progression-free survival $(P=0.02)$ and overall survival $(P=0.01)$. We then performed subgroup analysis based on $1 \mathrm{p} / 19 \mathrm{q}$ status. As $I D H$ mutation is regarded as an event preceding 1p/19q codeletion in oligodendroglial tumor formation, we focused the analysis on IDH mutants only. In 1p/19q-codeleted oligodendroglial tumors, patient age $\leq 45$ years showed longer progression-free survival $(P=0.0002)$, but tumor grade was not associated with survival. Tumors harboring CIC mutation exhibited worse overall survival compared with those with wild-type CIC $(P=0.01)$. In tumors with CIC deficiency or alteration, shorter progression-free survival was observed $(P=0.004 ; P=0.05)$. Moreover, loss of FUBP1 expression was significantly associated with unfavorable progression-free survival $(P=0.0003)$ and overall survival $(P=0.0004)$, whereas FUBP1 alteration was correlated with shorter progressionfree survival $(P=0.03)$. In tumors lacking $1 \mathrm{p} / 19 \mathrm{q}$ codeletion, low histologic grade and young patient age were significantly associated with favorable survival $(P=0.03 ; P=0.00004)$. No molecular markers showed association with survival in this group.

In multivariate analysis, Cox proportional-hazards model involved variables that showed statistical significance for progression-free survival and overall survival in univariate analysis, encompassing patient age at diagnosis, WHO grade, $1 \mathrm{p} / 19 \mathrm{q}$ codeletion, $C I C$ mutation, CIC expression, CIC alteration and FUBP1 expression. Patient of age $\leq 45$ years and low histologic tumor grade were identified as independent prognostic factors of favorable progression-free survival $\quad(\mathrm{HR}=0.085, \quad 95 \% \quad \mathrm{CI}=0.021-0.034$, $P=0.001 ; \mathrm{HR}=0.22,95 \% \mathrm{CI}=0.067-0.75, P=0.02$ ) and overall survival $(\mathrm{HR}=0.023,95 \% \mathrm{CI}=0.003-$ $0.17, P=0.0002 ; \mathrm{HR}=0.11,95 \% \mathrm{CI}=0.026-0.49$, $P=0.003$ ). 1p/19q Codeletion was also identified as an independent predictor of better overall survival $(\mathrm{HR}=0.24,95 \% \mathrm{CI}=0.065-0.84, P=0.03)$.

\section{Discussion}

This study determined the mutation incidences and expression patterns of $C I C$ and FUBP 1 and assessed the clinical relevance of these molecular markers in oligodendroglial tumors. Our study revealed that 


\begin{tabular}{|c|c|c|c|c|c|c|c|c|c|c|c|c|c|c|c|}
\hline \multirow[b]{2}{*}{ Variable } & \multicolumn{5}{|c|}{ All cases } & \multicolumn{5}{|c|}{$\begin{array}{l}\text { IDH mutation with } \\
1 p / 19 q \text { codeletion }\end{array}$} & \multicolumn{5}{|c|}{$\begin{array}{l}\text { IDH mutation with no } \\
1 p / 19 q \text { codeletion }\end{array}$} \\
\hline & $\mathrm{n}$ & $\begin{array}{l}\text { Median PFS } \\
\text { (months) }\end{array}$ & P-value & $\begin{array}{l}\text { Median OS } \\
\text { (months) }\end{array}$ & P-value & $\mathrm{n}$ & $\begin{array}{c}\text { Median } \\
\text { PFS (months) }\end{array}$ & P-value & $\begin{array}{c}\text { Median } \\
\text { OS (months) }\end{array}$ & P-value & $\mathrm{n}$ & $\begin{array}{l}\text { Median PFS } \\
\text { (months) }\end{array}$ & P-value & $\begin{array}{l}\text { Median OS } \\
\text { (months) }\end{array}$ & P-value \\
\hline & 44 & & & & & 17 & & & & & 23 & & & & \\
\hline \multicolumn{16}{|l|}{ Patient age (years) } \\
\hline $\begin{array}{l}\leq 45 \\
>45\end{array}$ & 26 & 9796.8 & 0.00002 & 142.1142 & 0.002 & 9 & 87.688 & 0.0002 & 159.3159 & 0.2 & 15 & 53.754 & 0.07 & 132.5133 & 0.00004 \\
\hline$>45$ & 18 & 2524.8 & & 86.486 & & 8 & 23.724 & & 116.9117 & & 8 & 24.825 & & 26.627 & \\
\hline \multicolumn{16}{|l|}{ Sex } \\
\hline Male & 25 & 4039.9 & 0.09 & 120.6121 & 0.2 & 9 & 43.243 & 0.2 & 122.3122 & 0.3 & 15 & 35 & 0.9 & 97.397 & 0.8 \\
\hline Female & 19 & 6968.5 & & 142.1142 & & 8 & 81.081 & & NR & & 8 & 33.934 & & 109.1109 & \\
\hline \multicolumn{16}{|l|}{ Tumor type } \\
\hline Oligodendroglioma & 24 & 5454.1 & 0.6 & 132.5133 & 0.3 & 13 & 50.951 & 0.3 & 159.3159 & 1 & 10 & 54.154 & 0.07 & 109.1109 & 0.4 \\
\hline Oligoastrocytoma & 20 & 3433.9 & & 120.6121 & & 4 & NR & & 116.9117 & & 13 & 26.627 & & 86.487 & \\
\hline \multicolumn{16}{|l|}{ Tumor grade } \\
\hline WHO grade II & 32 & 5453.7 & 0.06 & 141.5142 & 0.005 & 13 & 68.569 & 0.6 & NR & 0.1 & 15 & 53.754 & 0.07 & 120.6121 & 0.04 \\
\hline WHO grade III & 12 & 24.825 & & 109.1109 & & 4 & 56.857 & & 122.3122 & & 8 & 21.522 & & 37.237 & \\
\hline \multicolumn{16}{|l|}{ Extent of resection } \\
\hline Total & 8 & 43.243 & 0.2 & NR & 0.5 & 3 & 43.243 & 0.7 & NR & 0.8 & 2 & 52.653 & 0.6 & 26.627 & 0.7 \\
\hline Non-total & 28 & 52.653 & & 132.5133 & & 12 & 56.857 & & 159.3159 & & 15 & 26.627 & & 109.1109 & \\
\hline \multicolumn{16}{|l|}{ CIC mutation } \\
\hline Mutant & 15 & 52.653 & 0.7 & 116.9117 & 0.07 & 8 & 24.024 & 0.1 & 116.9117 & 0.01 & 7 & 26.627 & 0.6 & 109.1109 & 0.6 \\
\hline Wild-type & 21 & 35.035 & & 159.3159 & & 8 & 81.081 & & NR & & 11 & 54.154 & & 120.6121 & \\
\hline \multicolumn{16}{|l|}{ CIC expression } \\
\hline Negative & 16 & 27.327 & 0.03 & 109.1109 & 0.1 & 6 & 13.914 & 0.004 & 116.9117 & 0.1 & 9 & 35.035 & 0.8 & 109.1109 & 0.6 \\
\hline Positive & 28 & 68.569 & & 135.2135 & & 11 & 81.081 & & NR & & 14 & 33.934 & & 132.5133 & \\
\hline \multicolumn{16}{|l|}{ CIC alteration } \\
\hline Yes & 23 & 35.035 & 0.1 & 109.1109 & 0.05 & 9 & 24.024 & 0.05 & 122.3122 & 0.06 & 13 & 52.653 & 0.9 & 97.397 & 0.2 \\
\hline No & 17 & 43.243 & & 159.3159 & & 7 & 81.081 & & NR & & 9 & 25.526 & & 135.2135 & \\
\hline \multicolumn{16}{|l|}{ FUBP1 mutation } \\
\hline Mutant & 5 & 13.914 & 0.09 & 109.1109 & 0.6 & 4 & 13.914 & 0.08 & 159.3159 & 0.5 & 1 & 31.732 & 0.7 & 109.1109 & 0.6 \\
\hline Wild-type & 29 & 50.951 & & 135.2135 & & 12 & 56.857 & & 116.9117 & & 15 & 33.934 & & 132.5133 & \\
\hline \multicolumn{16}{|l|}{ FUBP1 expression } \\
\hline Negative & 7 & 19.620 & 0.02 & 47.748 & 0.01 & 4 & 13.914 & 0.0003 & 47.748 & 0.0004 & 3 & 31.732 & 0.7 & 109.1109 & 0.7 \\
\hline Positive & 37 & 54.154 & & 135.2135 & & 13 & 81.081 & & NR & & 20 & 35.035 & & 97.397 & \\
\hline \multicolumn{16}{|l|}{ FUBP1 alteration } \\
\hline Yes & 8 & 23.724 & 0.1 & 109.1109 & 0.2 & 5 & 13.914 & 0.03 & 47.748 & 0.1 & 3 & 31.732 & 0.7 & 109.1109 & 0.7 \\
\hline No & 28 & 50.951 & & 142.1142 & & 11 & 81.081 & & 159.3159 & & 15 & 33.934 & & 132.5133 & \\
\hline \multicolumn{16}{|l|}{$1 p / 19 q$ Codeletion } \\
\hline Yes & 17 & 56.857 & 0.4 & 159.3159 & 0.05 & & & & & & & & & & \\
\hline No & 27 & 39.940 & & 109.1109 & & & & & & & & & & & \\
\hline \multicolumn{16}{|l|}{ IDH mutation } \\
\hline $\begin{array}{l}\text { Yes } \\
\text { No }\end{array}$ & 40 & 52.653 & 0.4 & 132.5133 & 0.7 & & & & & & & & & & \\
\hline No & 4 & 39.940 & & 91.692 & & & & & & & & & & & \\
\hline
\end{tabular}

Abbreviations: $n$, number of patients; NR, median value not yet reached; OS, overall survival; PFS, progression-free survival. 
CIC and FUBP1 mutations were detectable in $47 \%$ and $16 \%$ of oligodendroglial tumors, respectively. Combining our results with the published data, the overall rates of CIC and FUBP1 mutation were $46 \%$ $(108 / 235)$ and $20 \%(28 / 143)$ in oligodendrogliomas and $17 \%(22 / 127)$ and $6 \%(6 / 95)$ in oligoastrocytomas. ${ }^{12-15}$ CIC mutations were found predominantly in tumors carrying $1 \mathrm{p} / 19 \mathrm{q}$ codeletion or partial $19 \mathrm{q}$ deletion and were associated with absent CIC expression. FUBP1 mutation was frequently detected concurrently with $C I C$ mutation and was associated with absent FUBP1 expression. These results suggest that inactivation of $C I C$ and FUBP1, through mutation and chromosome loss, contribute to the development of oligodendroglial tumors.

$C I C$ was first identified as a downstream repressor of terminal genes in receptor tyrosine kinase (RTK) pathway in regulating embryonic terminal patterning of Drosophila. ${ }^{20}$ It has been shown that RTK signaling suppresses function of CIC protein and abolishes its transcriptional repression and leads to expression of genes normally repressed by CIC protein. ${ }^{20,21}$ Two highly conserved functional domains in CIC protein were responsible for the transcriptional repression-the HMG box domain and the C-terminal motif $\mathrm{C} 1 .^{20-22}$ Of note, $70 \%$ $(16 / 23)$ of CIC mutations in our cohort were located in exon 5, 6 (encode HMG box) and 20 (encode C1 motif). The functional consequence of CIC mutations identified in oligodendroglial tumors is unclear and further investigation is needed to understand the role CIC has in oligodendroglial tumor development. The protein encoded by FUBP1 was pivotal for cellular proliferation. ${ }^{23}$ High expression of FUBP1 was found in mouse and chicken embryonic brain and was developmentally regulated. ${ }^{24}$ FUBP1 bound to FUSE (far upstream element) of proto-oncogene $c$-myc, ${ }^{25}$ together with interaction with FIR (FBPinteracting repressor), formed a regulatory system for fine-tuning $C-m y c$ transcription. ${ }^{26}$ Disruption of $c$-myc transcriptional regulation due to FUBP1 mutation probably contributes to oncogenesis of oligodendroglial tumors. Further study will be needed to elucidate the role FUBP1 involved in the pathogenesis of oligodendroglial tumors.

The $1 \mathrm{p} / 19 \mathrm{q}$ status separates oligodendroglial tumors into two groups of distinct clinical features. Oligodendroglial tumors without $1 \mathrm{p} / 19 \mathrm{q}$ codeletion show poor prognosis and have heterogeneous genotypes. Genetic alterations such as EGFR amplification, $7 p$ gain and $21 q$ loss have been associated with shorter survival. ${ }^{27,28}$ Tumors carrying $1 \mathrm{p} / 19 \mathrm{q}$ codeletion are correlated with $I D H$ mutation, proneural gene expression pattern and a hypermethylated phenotype CIMP + and are linked to favorable prognosis. ${ }^{29-31}$ However, a fraction of patients with $1 p / 19 q$ codeletion exhibit poor survival, despite combined radiochemotherapy.,6 Identification of prognostic markers that can substratify $1 \mathrm{p} / 19 \mathrm{q}$ codeletion will improve clinical management of oligodendroglial tumors.
The discovery of $C I C$ and FUBP1 mutations has turned these markers into one of the hallmarks of oligodendroglial tumors. 1p/19q-Codeleted oligodendroglial tumors can be stratified into two groups either with or without CIC and FUBP1 mutations. ${ }^{32}$ The prognostic significance of CIC mutation had been examined by two research teams. Yip et $a l^{13}$ found no survival difference between 20 CIC-mutant and 23 CIC-wild-type oligodendroglioma patients. Jiao et $a l^{15}$ classified gliomas by genetic alterations and defined the 'I-CF' group as carrying IDH1 mutation in combination with $1 \mathrm{p} / 19 \mathrm{q}$ codeletion, CIC mutation or FUBP1 mutation. Majority of I-CF gliomas were oligodendroglial tumors. Their results showed no survival difference between 16 I-CF gliomas with CIC mutation and 10 I-CF gliomas without CIC mutation. ${ }^{15}$ In our series of 16 cases carrying $1 p / 19 q$ codeletion, 8 had CIC mutations and 8 without. We cautiously examined our data and revealed that tumors with CIC mutation exhibited unfavorable survival compared with those without mutation $(P=0.01)$. No association of $C I C$ mutation with survival was observed in the whole series and in tumors lacking 1p/19q codeletion. Results from our series suggest that CIC mutation is a potential prognostic marker in oligodendroglial tumors carrying $1 \mathrm{p} / 19 \mathrm{q}$ codeletion. Further investigation with a large number of samples is required to define the prognostic significance of CIC mutation in oligodendroglial tumors.

A major finding of this study was the shorter progression-free survival seen in tumors with absent CIC or FUBP1 expression at initial diagnosis than those expressing either protein (CIC 27 months vs 69 months, $P=0.03$; FUBP1 20 months vs 54 months, $P=0.02)$. Such associations became more significant in tumors with $1 p / 19 q$ codeletion (CIC 14 vs 81 months, $P=0.004$; FUBP1 14 vs 81 months, $P=0.0003)$. The progression-free survival for $1 \mathrm{p} /$ 19q-non-deleted tumors with and without CIC or FUBP1 expression was similar. These data suggest that loss of CIC or FUBP1 expression is a potential marker of shorter time to recurrence or tumor progression, particularly in tumors carrying $1 \mathrm{p} / 19 \mathrm{q}$ codeletion. A recent study has shown that oligodendroglial tumors concurrently carrying $1 \mathrm{p} / 19 \mathrm{q}$ codeletion and polysomy 1 and/or 19q showed earlier recurrence than those without polysomy. ${ }^{33}$ It would be of interest to investigate whether absent CIC or FUBP1 expression in combination with polysomy has a greater power in predicting recurrence in tumors with $1 \mathrm{p} / 19 \mathrm{q}$ codeletion.

The somatic changes identified in the FUBP1 gene were predicted to be deleterious to FUBP1 protein structure or function. The anti-FUBP1 antibody used was able to identify almost all mutations, indicating that FUBP1 expression can be a surrogate marker for mutation. For CIC, absent expression was readily detected in tumors with frameshift indels and $1 \mathrm{p} / 19 \mathrm{q}$ codeletion. A fraction of tumors carrying 
missense mutations and small inframe deletions exhibited CIC expression. These mutations were predominantly located on exons corresponding to the HMG box (exons 5 and 6) and the $\mathrm{C} 1$ motif (exon 20). Of note was the identification of four tumors with wild-type CIC sequence but absent CIC expression. All of these cases had short progression-free survival, ranging from 13 to 35 months. We propose that combined immunohistochemistry and mutation screen on HMG box and C1 motif is a simple and fast approach to disclosing inactivated $C I C$.

In conclusion, young patient age at diagnosis, low histologic tumor grade and $1 \mathrm{p} / 19 \mathrm{q}$ codeletion are independent predictors of favorable outcome in oligodendroglial tumors. In tumors carrying $1 \mathrm{p} / 19 \mathrm{q}$ codeletion, presence of $C I C$ mutation is a potential marker of survival and null expression of CIC or FUBP1 is a potential marker of shorter time to recurrence. Further investigation is required to understand the role $C I C$ and $F U B P 1$ has in $1 \mathrm{p} / 19 \mathrm{q}-$ codeleted oligodendroglial tumors.

\section{Acknowledgements}

This study was supported by the National Science Foundation of China (Grant No. 81172412).

\section{Disclosure/conflict of interest}

The authors declare no conflict of interest.

\section{References}

1 van den Bent MJ, Reni M, Gatta G, et al. Oligodendroglioma. Crit Rev Oncol Hematol 2008;66: 262-272.

2 Reifenberger J, Reifenberger G, Liu L, et al. Molecular genetic analysis of oligodendroglial tumors shows preferential allelic deletions on $19 q$ and $1 p$. Am J Pathol 1994;145:1175-1190.

3 Cairncross JG, Ueki K, Zlatescu MC, et al. Specific genetic predictors of chemotherapeutic response and survival in patients with anaplastic oligodendrogliomas. J Natl Cancer Inst 1998;90:1473-1479.

4 Smith JS, Perry A, Borell TJ, et al. Alterations of chromosome arms $1 p$ and $19 q$ as predictors of survival in oligodendrogliomas, astrocytomas, and mixed oligoastrocytomas. J Clin Oncol 2000;18: 636-645.

5 van den Bent MJ, Brandes AA, Taphoorn MJ, et al. Adjuvant procarbazine, lomustine, and vincristine chemotherapy in newly diagnosed anaplastic oligodendroglioma: long-term follow-up of EORTC brain tumor group study 26951. J Clin Oncol 2013;31: 344-350.

6 Cairncross G, Wang M, Shaw E, et al. Phase III trial of chemoradiotherapy for anaplastic oligodendroglioma: long-term results of RTOG 9402. J Clin Oncol 2013; 31:337-343.
7 Balss J, Meyer J, Mueller W, et al. Analysis of the IDH1 codon 132 mutation in brain tumors. Acta Neuropathol 2008;116:597-602.

8 Watanabe T, Nobusawa S, Kleihues $\mathrm{P}$, et al. IDH1 mutations are early events in the development of astrocytomas and oligodendrogliomas. Am J Pathol 2009;174:1149-1153.

9 van den Bent MJ, Dubbink HJ, Marie Y, et al. IDH1 and IDH2 mutations are prognostic but not predictive for outcome in anaplastic oligodendroglial tumors: a report of the European Organization for Research and Treatment of Cancer Brain Tumor Group. Clin Cancer Res 2010;16:1597-1604.

10 Griffin CA, Burger P, Morsberger L, et al. Identification of $\operatorname{der}(1 ; 19)(q 10 ; p 10)$ in five oligodendrogliomas suggests mechanism of concurrent $1 p$ and $19 q$ loss. J Neuropathol Exp Neurol 2006;65:988-994.

11 Jenkins RB, Blair $H$, Ballman $\mathrm{KV}$, et al. A $\mathrm{t}(1 ; 19)$ (q10;p10) mediates the combined deletions of $1 p$ and $19 \mathrm{q}$ and predicts a better prognosis of patients with oligodendroglioma. Cancer Res 2006;66:9852-9861.

12 Bettegowda C, Agrawal N, Jiao Y, et al. Mutations in CIC and FUBP1 contribute to human oligodendroglioma. Science 2011;333:1453-1455.

13 Yip S, Butterfield YS, Morozova O, et al. Concurrent CIC mutations, IDH mutations, and 1p/19q loss distinguish oligodendrogliomas from other cancers. J Pathol 2012;226:7-16.

14 Sahm F, Koelsche C, Meyer J, et al. CIC and FUBP1 mutations in oligodendrogliomas, oligoastrocytomas and astrocytomas. Acta Neuropathol 2012;123: 853-860.

15 Jiao Y, Killela PJ, Reitman ZJ, et al. Frequent ATRX, $C I C$, and FUBP1 mutations refine the classification of malignant gliomas. Oncotarget 2012;3:709-722.

16 Reifenberger G, Kros JM, Louis DN, et al. Oligodendroglioma. In: Louis DN, Ohgaki H, Wiestler OD, Cavenee WK (eds). WHO Classification of Tumours of the Central Nervous System. International Agency for Research on Cancer: Lyon, France, 2007, pp 54-67.

17 Dong Z, Pang JS, Ng MH, et al. Identification of two contiguous minimally deleted regions on chromosome 1p36.31-p36.32 in oligodendroglial tumours. $\mathrm{Br} \mathrm{J}$ Cancer 2004;91:1105-1111.

18 Adzhubei IA, Schmidt S, Peshkin L, et al. A method and server for predicting damaging missense mutations. Nat Methods 2010;7:248-249.

19 Tseng AS, Tapon N, Kanda H, et al. Capicua regulates cell proliferation downstream of the receptor tyrosine kinase/ras signaling pathway. Curr Biol 2007;17: 728-733.

20 Jiménez G, Guichet A, Ephrussi A, Casanova J. Relief of gene repression by torso RTK signaling: role of capicua in Drosophila terminal and dorsoventral patterning. Genes Dev 2000;14:224-231.

21 Astigarraga S, Grossman R, Díaz-Delfín J, et al. A MAPK docking site is critical for downregulation of Capicua by Torso and EGFR RTK signaling. EMBO J 2007;26:668-677.

22 Lee CJ, Chan WI, Cheung M, et al. CIC, a member of a novel subfamily of the HMG-box superfamily, is transiently expressed in developing granule neurons. Brain Res Mol Brain Res 2002;106:151-156.

23 He L, Liu J, Collins I, et al. Loss of FBP function arrests cellular proliferation and extinguishes c-myc expression. EMBO J 2000;19:1034-1044. 
24 Wang X, Avigan M, Norgren RB Jr. FUSE-binding protein is developmentally regulated and is highly expressed in mouse and chicken embryonic brain. Neurosci Lett 1998;252:191-194.

25 Duncan R, Bazar L, Michelotti G, et al. A sequencespecific, single-strand binding protein activates the far upstream element of c-myc and defines a new DNAbinding motif. Genes Dev 1994;8:465-480.

26 Hsiao HH, Nath A, Lin CY, et al. Quantitative characterization of the interactions among c-myc transcriptional regulators FUSE, FBP, and FIR Biochemistry 2010;49:4620-4634.

27 Trost D, Ehrler M, Fimmers R, et al. Identification of genomic aberrations associated with shorter overall survival in patients with oligodendroglial tumors. Int J Cancer 2007;120:2368-2376.

28 Idbaih A, Dalmasso C, Kouwenhoven M, et al. Genomic aberrations associated with outcome in anaplastic oligodendroglial tumors treated within the EORTC phase III trial 26951. J Neurooncol 2011;103:221-230.
29 Ducray F, Idbaih A, de Reyniès A, et al. Anaplastic oligodendrogliomas with 1p19q codeletion have a proneural gene expression profile. Mol Cancer 2008; 7:41.

30 Cooper LA, Gutman DA, Long Q, et al. The proneural molecular signature is enriched in oligodendrogliomas and predicts improved survival among diffuse gliomas. PLoS One 2010;5:e12548.

31 van den Bent MJ, Gravendeel LA, Gorlia T, et al. A hypermethylated phenotype is a better predictor of survival than MGMT methylation in anaplastic oligodendroglial brain tumors: a report from EORTC study 26951. Clin Cancer Res 2011;17:7148-7155.

32 Alentorn A, Sanson M, Idbaih A. Oligodendrogliomas: new insights from the genetics and perspectives. Curr Opin Oncol 2012;24:687-693.

33 Snuderl M, Eichler AF, Ligon KL, et al. Polysomy for chromosomes 1 and 19 predicts earlier recurrence in anaplastic oligodendrogliomas with concurrent 1p/19q loss. Clin Cancer Res 2009;15:6430-6437.

Supplementary Information accompanies the paper on Modern Pathology website (http://www.nature.com/ modpathol) 